\title{
Single-Carrier Frequency-Domain Equalizer with Multi-Antenna Transmit Diversity
}

\author{
Sunil Shankar Mane ${ }^{1}$, Dr. S. L. Lahudkar ${ }^{2}$ \\ Student, E\&TC Engg, JSPM's ICOER, Pune, India ${ }^{1}$ \\ Asst. Prof., E\&TC Engg, JSPM's ICOER, Pune, India ${ }^{2}$
}

\begin{abstract}
Single-carrier (SC) block transmission with cyclic prefix (CP) is a method with several advantages that has been incorporated into standards. This paper has analyzed the performance of multi-antenna SC-FDE under Alamouti signaling and cyclic-delay diversity (CDD). Our analysis shows that the characteristic of diversity it is depends on data block length and data transmission rate as well as on the channel memory and antenna configuration. At higher rates their diversity diminishes and full diversity is available to both CDD and Alamouti signalling below a certain rate threshold. From our investigation we say that at high rates Alamouti signalling provides twice the diversity of SISO SC-FDE, while the diversity of the SISO SC-FDE under the CDD diversity degenerates.
\end{abstract}

Keywords: Cyclic Prefixes, Cyclic Delay Diversity, Alamouti Signalling, Single-Carrier.

\section{INTRODUCTION}

Orthogonal frequency division multiplexing (OFDM) is an effective technique that combats this effect by converting a frequency selective fading channel into a set of nearly flat narrowband orthogonal fading channels, thereby solving equalization at the receiver side. SINGLECARRIER Frequency Domain Equalization (SCFDE) is an alternative to OFDM that avoids several OFDM drawbacks, including the high sensitivity to carrier frequency offset and peak-to-average power ratio.

This article discusses an alternative approach based on more traditional single-carrier (SC) modulation methods. We had shown that when it combined with FDE, this SC modulation approach delivers performance with essentially the same overall complexity similar to OFDM. In addition, $\mathrm{SC}$ modulation uses a single carrier so the peak-to-average transmitted power ratio for SC-modulated signals is smaller. This in turn means that the power amplifier of an SC transmitter requires a smaller linear range to support a given average power. So we can use the use of a cheaper power amplifier than a comparable OFDM system; and this is a benefit of some importance, since the power amplifier can be one of the more costly components in a consumer broadband wireless transceiver.

\section{SCOPE}

In this paper, we analyze the performance of SC-FDE in conjunction with either cyclic delay diversity (CDD) or Alamouti signaling, fully characterizing the diversity as a function of transmission-block length, number of antennas data rate and channel memory. In the process, we obtain a threshold rate (as a function of data-block length, channel memory, and number of antennas) below which the full spatial-temporal diversity is achieved, while at higher rates the diversity of both schemes diminishes. Our analysis shows that at high rates Alamouti signaling provides twice the diversity of SISO SCFDE, while the CDD diversity degenerates to the diversity of the SISO SC-FDE.

\section{CYCLIC PREFIX TRANSMISSION}

We consider a frequency selective quasi-static wireless fading channel. For this intersymbol interference (ISI) channel the equivalent baseband model is given by a multipath model with $v+1$ paths. The channel coefficients and the channel vector is denoted by $h=[\mathrm{h} 0 \ldots \ldots . \mathrm{h} v]$ are assumed independent and identically distributed $\sim \mathrm{CN}(0$, 1). We assume a block-fading model where the channel is fixed over the transmission block. A cyclic-prefix (CP) with length at least $v$ is inserted at the beginning of each data-block of length $\mathrm{L}$ to remove the inter-block interference at the receiver. The $\mathrm{CP}$ also transforms linear convolution into circular convolution and thus permits channel diagonalization. The I/O system model for a block transmission scheme with length- $v \mathrm{CP}$ is

$$
\begin{gathered}
\mathbf{y}=\sqrt{\rho \mathbf{I x}}+\mathbf{n} \\
\mathbf{y}=\sqrt{\rho} \mathbf{H U}_{c p} \mathbf{s}+\mathbf{n}=\sqrt{\rho} \mathbf{H}_{\mathbf{e}} \mathbf{s}+\mathbf{n}
\end{gathered}
$$

In single-carrier frequency domain equalizer (SC-FDE), the DFT/IDFT operation is performed at the receiver. This operation in the SC-FDE diagonalizes the channel thus a single-tap equalizer can be used to reduce the complexity of equalization. The DFT of Equation (2) is

$$
\mathbf{Y}=\mathbf{Q} \mathbf{y}=\sqrt{\rho} \Lambda \mathbf{S}+\mathbf{N}
$$

Where N, S and Y are the DFT of the noise, transmitted and received vectors respectively.

In [3] the linear MMSE receiver is analyzed for SC-FDE, as we are studying later in this paper and therefore we review it here briefly. In [3] the received signal after equalization is given by

$$
\tilde{Y}=W y=\sqrt{\rho} W H_{e} s+W n
$$

and subject to this model it was shown that MMSE SCFDE can achieve full diversity for certain values of block length and operating rate $\mathrm{R} \mathrm{b} / \mathrm{s} / \mathrm{Hz}$.

The process of developing this result was as follows. 
The analysis performed in [3] consists of two main steps. system with CDD. In vector form, the received signal can We first characterize the outage probability. Then the be written as lower and upper bounds on the error probability via outage are provided. It is shown that these two bounds are tight and thus the diversity is fully characterized. This type of approach was first proposed in [12] due to the intractability of the direct pair wise error probability (PEP) analysis for many MIMO architectures.

The diversity of the MMSE SC-FDE is [3]
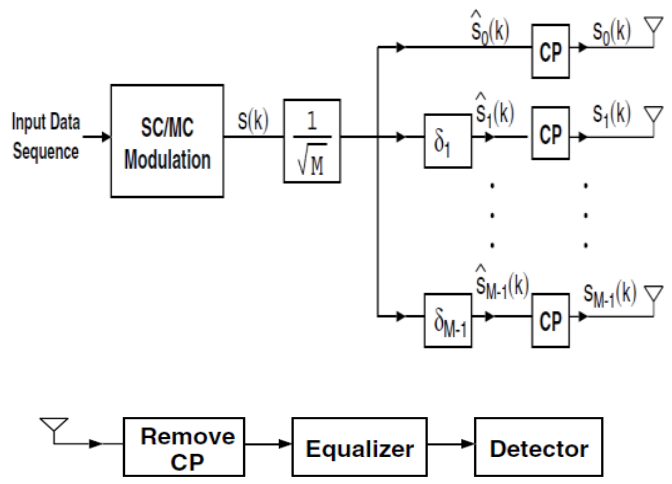

$$
d_{\text {out }}=\left\{\begin{array}{cc}
v+1 & R \leq \log \frac{L}{v} \\
2^{-R} L & R>\log \frac{L}{v}
\end{array}\right.
$$

Fig.1. Single- carrier and multicarrier MISO system with transmitter sided CDD scheme and the proposed MMSE receiver.

\section{CYCLIC DEAY DIVERSITY}

One common transmit diversity technique used for single carrier and multicarrier systems is antenna delay diversity, which can take the form of time delay, cyclic delay and phase delay [13], [14]. Among them, cyclic delay diversity (CDD) is more widely adopted for single carrier and multicarrier applications as CDD can be applied to any number of transmit antennas without any rate loss or change in the receiver structure [14]-[16]. In this section we show that linear MMSE receivers can achieve the maximal spatio-temporal diversity provided that the equalizer and the cyclic delay taps are properly designed.

1) System Model: Consider a MISO system with $M$ transmit antennas and a block fading channel model where the channel remains unchanged during the block transmission. The channel impulse response from the transmit antenna I to the received antenna is given by hi $=$ $[$ hi, $0, \ldots$, hi,vi ] with channel memory length denoted by vi. We also define $v=$ maxi vi. We adopt the system model of [14]. The model is shown in Figure 1 which displays the front end of a single carrier and multicarrier MISO

$$
Y=\sum_{i=0}^{M-1} \sqrt{\rho} H_{i} \hat{S}_{i}+n
$$

Where $\mathrm{Hi}$ is an $\mathrm{L} \times \mathrm{L}$ circulant channel matrix whose first row is $[h i, 0, \ldots$, hi,vi $, 0, \ldots, 0]$, is the $\mathrm{L} \times 1$ transmitted datablock (without the $\mathrm{CP}$ ) from transmit antenna i. CDD converts the MISO channel into a SISO channel with increased channel selectivity. The model can be written as [15]

$$
Y=\sqrt{\rho H_{c i r}} S+n=\sqrt{\rho} Q^{H} \Lambda Q s+n
$$

where Hcir is $\mathrm{L} \times \mathrm{L}$ circulant matrix, $\mathrm{s}$ is the $\mathrm{L} \times 1$ modulated symbols (cf. Figure 1), Q is the $\mathrm{L} \times \mathrm{L}$ normalized DFT matrix, and $\Lambda$ is a diagonal matrix whose diagonal entries are the DFT point of the first row of Hcir.

The selection of the delay samples $\{\delta i\}$ and its impact on the data rate, the signal-to-interference-and-noise ratio (SINR) and maximum achievable diversity is studied in [15], [17]. While the CP length is independent of $\delta \mathrm{i}$, it must be no less than the maximum channel delay spread $v$ [17]. Also for the receiver to exploit the full diversity the delays can be chosen as $\delta \mathrm{i}>\delta \mathrm{i}-1+v[15]$ or simply $\delta \mathrm{i}=$ i $\delta$ with $\delta>v$

2) Diversity Analysis of MMSE Receiver: We first consider the case where $v i=v=0 \forall i$ (i.e. flat MISO channel) and the symbol delays $\delta \mathrm{i}=\mathrm{i}$. In this case, the system model is equivalent to a SISO ISI channel under $\mathrm{CP}$ transmission. If the equalizer is designed according to Hcir, it is known that in the SISO ISI CP transmission a rate-dependent diversity is observed [3], and due to equivalence of channel models this result can be directly lifted to the flat MISO CDD system. This result will be extended to the general case of the multipath MISO channel under CDD.

Lemma 1: Consider the $\mathrm{M} \times 1$ MISO flat-fading channel. The diversity of the MMSE receiver under uncoded CDD transmission and L data-blocks is given by

$$
d_{\text {out }}=\left\{\begin{array}{cc}
M & R \leq \log \frac{L}{(M-1)} \\
2^{-R} L+1 & R>\log \frac{L}{(M-1)}
\end{array}\right.
$$

Remark 1: Note that the uncoded CP systems can suffer from loss of multipath diversity [18]. However if a linear receiver is used and the system parameters (rate, transmit antenna delays, and FFT block length) are appropriately designed, Lemma 1 indicates that the maximum diversity can be achieved. Specifically, the maximum diversity is achieved when

$$
\left\lfloor L 2^{-R}\right\rfloor+1 \geq M
$$


For any given values of $\mathrm{R}$ and $\mathrm{M}$, the data block length $\mathrm{L}$ can be chosen such that $R \leq R_{\text {th }}$. Therefore if we have flexibility in assigning data block length, maximum diversity can always be achieved.

Remark 2: The developments in this paper do not depend on whether the equalization matrix $\mathrm{W}$ is multiplied by the received data in the time domain, or is applied in the frequency domain (via FFT/IFFT). Both approaches lead to the same SINR and outage. Therefore, the results of this paper are valid for single-carrier systems regardless of whether the receiver operates in the time domain or frequency domain.

We now consider the second case: the frequency selective channel, i.e., $v \mathrm{i}=0$. The delay taps are chosen such that $\delta \mathrm{i}=\mathrm{ni} \delta$ with $\delta>v$ and $\{\mathrm{ni}\}$ are distinct integers, and the block length $\mathrm{L}$ is chosen such that the transmitted blocks si are distinct. Notice that the condition on the block length guarantees that the channel coefficients seen at the receiver are independent. Thus the delay taps and the block length $\mathrm{L}$ must be chosen to satisfy two conditions

$$
\begin{aligned}
n_{i} \delta_{0} \bmod L & =n_{j} \delta_{0} \bmod L \quad \forall i \neq j \\
L & \geqslant M(\nu+1) .
\end{aligned}
$$

We now consider the following case $1: v \mathrm{i}=v \forall \mathrm{i}, \delta \mathrm{i}=\mathrm{i}(v+$ 1). It can be shown that this system is equivalent to (15) where the variable $\mathrm{M}$ in (15) is replaced by $\mathrm{M}(v+1)$. Thus we obtain the diversity

$$
d_{\text {out }}=\left\{\begin{array}{cc}
M & R \leq \log \frac{L}{(M(v+1)-1)} \\
2^{-R} L+1 & R>\log \frac{L}{(M(v+1)-1)}
\end{array}\right.
$$

Other choices of $\{\delta i\}$ that satisfy (18) exist. Each of these choices yields a new Hcir whose first row is a permutation of the first row of Hcir under $\delta \mathrm{i}=\mathrm{i}(v+1)$. We thus have $\mathrm{L}$ ! different choices for the set $\{\delta \mathrm{i}\}$. Since these circulant matrices have different structures, the diversity analysis of [3] does not directly follow. We study the diversity of these systems and show that the L!-1 remaining choices $\{\delta \mathrm{i}\}$ yield the same diversity as shown in (20).

$$
P_{\text {out }} \doteq \mathbb{P}\left(\sum_{k=1}^{L} \frac{1}{1+\rho\left|\lambda_{k}\right|^{2}}>L 2^{-R}\right)
$$

\section{ALAMOUTI SIGNALING}

The Alamouti method of space-time signaling can also be characterized as a transmit diversity scheme. Unlike the CDD system, our analysis shows that Alamouti signaling preserves the transmit diversity and thus provides a larger diversity gain compared with the CDD scheme above a rate threshold Rth. We consider SC block transmission over an additive-noise frequency-selective channel with memory $v$, similar to [5]. The model supports a $2 \times 1$ system and can be extended to $2 \times \mathrm{N}$ system.

Each data-block of length $\mathrm{L}$ is appended with a $\mathrm{CP}$ of length $v$ to eliminate interblock interference. $\mathrm{x}(\mathrm{k}) \mathrm{I}(\mathrm{n})$ denotes the symbol $\mathrm{n}$ of the transmitted block $\mathrm{k}$ from antenna i. At even time slots, pairs of length-N blocks $\mathrm{x}(\mathrm{k}) 1(\mathrm{n})$ and $\mathrm{x}(\mathrm{k}) 2(\mathrm{n})$ are generated. The transmission scheme proposed by [5] is

$$
\begin{aligned}
& x_{1}^{(k+1)}(n)=-x *_{2}^{(k)}((-n) N) \\
& x_{2}^{(k+1)}(n)=-x *_{1}^{*(k)}((-n) N)
\end{aligned}
$$

for $\mathrm{n}=0,1, \ldots, \mathrm{N}-1$ and $\mathrm{k}=0,2,4 \ldots$, and $*$ denotes conjugate. A length- $v \mathrm{CP}$ is added to each transmitted block. The total transmit power is divided equally in between the antennas. In Figure 2, the transmission scheme is shown.

The received blocks at time $\mathrm{k}$ and $\mathrm{k}+1$ are given by

$$
Y^{(j)}=\sqrt{\rho} H_{1}^{(j)} x_{1}^{(j)}+\sqrt{\rho} H_{2}^{(j)} x_{2}^{(j)}+n^{(j)} \quad \text { for } j=k, k+1
$$

Where $Y(j), X(j)$ and $N(j)$ are the DFT vectors of $y(j), x(j)$ and $n(j)$ respectively, and $\Lambda \mathrm{i}($ for $\mathrm{i}=1,2)$ are diagonal matrices containing the DFT coefficients of the channel impulse responses. Using (33) and assuming the channels are fixed over two consecutive blocks (indexed by $\mathrm{k}$ and $\mathrm{k}$ +1 ), it can be shown that

$$
\mathbf{Y} \triangleq\left(\begin{array}{c}
\mathbf{Y}^{(k)} \\
\mathbf{Y}^{*(k+1)}
\end{array}\right)=\underbrace{\left(\begin{array}{cc}
\Lambda_{1} & \Lambda_{2} \\
\Lambda_{2}^{*} & -\Lambda_{1}^{*}
\end{array}\right)}_{\underline{\underline{\underline{\beta}} \Lambda}}\left(\begin{array}{c}
\sqrt{\rho} \mathbf{X}_{1}^{(k)} \\
\sqrt{\rho} \mathbf{X}_{2}^{*(k)}
\end{array}\right)+\left(\begin{array}{c}
\mathbf{N}^{(k)} \\
\mathbf{N}^{*(k+1)}
\end{array}\right) .
$$

By multiplying both sides of (34) by the orthogonal matrix $\Lambda^{*}$ defined in (34)

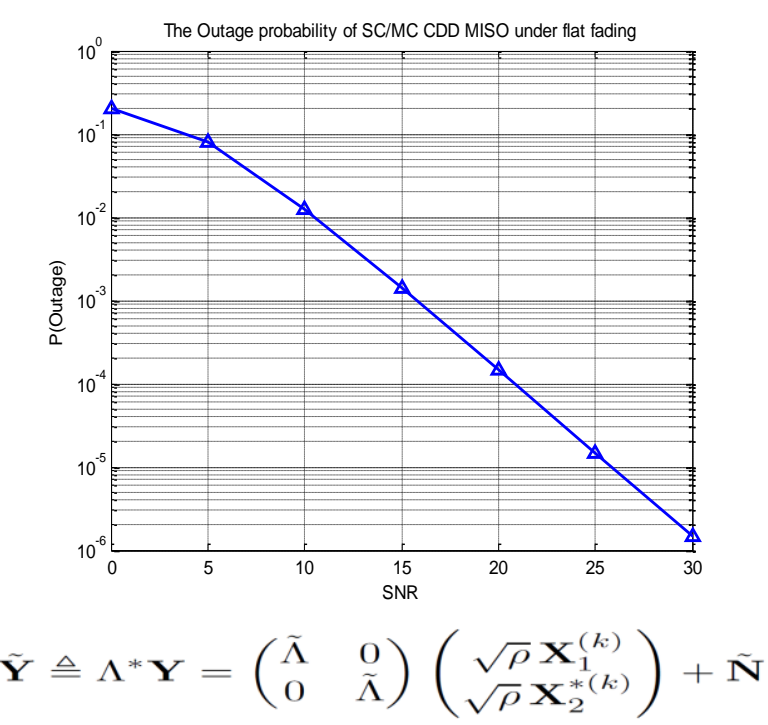

\section{SIMULATION RESULTS}

Fig. 2. The Outage probability of SC/MC CDD MISO under flat fading with three transmit antennas and $\mathrm{R}=2$ $\mathrm{b} / \mathrm{s} / \mathrm{Hz}$.

Figure 2 shows under various choices of the cyclic delay taps, the outage probability Pout for the equivalent model of the MMSE receiver in the CDD CP MISO flat fading channel with 3 transmit antennas. In this case, the MMSE diversity is two (as predicted from (20)) since this rate is 


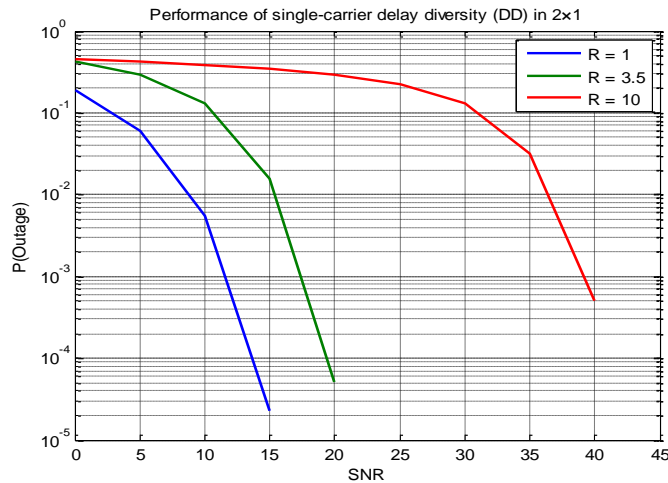

greater than Rth given by (21). The rate is $\mathrm{R}=2 \mathrm{~b} / \mathrm{s} / \mathrm{Hz}$ and $\mathrm{L}=5$. Fig. 3. Performance of single-carrier delay diversity (DD) and cyclic delay diversity (CDD) in $2 \times 1$ MISO under flat fading and $\mathrm{R}=1,3.5$, and $10 \mathrm{~b} / \mathrm{s} / \mathrm{Hz}$.

Figure 3 compares CDD-CP and DD-without-CP systems in a $2 \times 1$ MISO flat fading channel. The latter system is equivalent to zero-padding transmission over a SISO ISI channel with three channel coefficients and thus achieves the full diversity for all rates [8]. However, the CDD CPsystem only achieves full diversity for the rates that satisfy (21).

\section{CONCLUSION}

This paper analyzes the single-carrier frequency domain equalizer (SC-FDE) for two common transmit diversity schemes: cyclic delay diversity (CDD) and Alamouti signalling. We characterize the diversity for both schemes at all spectral efficiencies. In the process, we obtain a threshold rate (as a function of data-block length, channel memory, and number of antennas) below which the full spatial-temporal diversity is achieved. Our investigation shows that at high rates the CDD diversity degenerates to the diversity of the SISO SCFDE, while Alamouti signalling provides twice the diversity of SISO SC-FDE.

\section{ACKNOWLEDGMENT}

The heading of the Acknowledgment section and the References section must not be numbered. Causal Productions wishes to acknowledge Michael Shell and other contributors for developing and maintaining the IEEE LaTeX style files which have been used in the preparation of this template.

\section{REFERENCES}

[1] F. Pancaldi, G. Vitetta, R. Kalbasi, N. Al-Dhahir, M. Uysal, and H. Mheidat, "Single-carrier frequency domain equalization," IEEE Signal Process. Mag., vol. 25, no. 5, pp. 37-56, Sept. 2008.

[2] D. Falconer, S. Ariyavisitakul, A. Benyamin-Seeyar, and B. Eidson "Frequency domain equalization for single-carrier broadband wireless systems," IEEE Commun. Mag., vol. 40, no. 4, pp. 58-66, Apr. 2002.

[3] A. Tajer and A. Nosratinia, "Diversity order in ISI channels with singlecarrier frequency-domain equalizer," IEEE Trans. Wireless Commun., vol. 9, no. 3, pp. 1022-1032, Mar. 2010.

[4] A. Hesham Mehana and A. Nosratinia, "The diversity of MMSE receiver over frequency-selective MIMO channel," in Proc. 2011 IEEE ISIT.
[5] N. Al-Dhahir, "Single-carrier frequency-domain equalization for spacetime block-coded transmissions over frequency-selective fading channels,'IEEE Commun. Lett., vol. 5, no. 7, pp. 304-306, July 2001.

[6] Z. Wang and G. Giannakis, "Linearly precoded or coded OFDM against wireless channel fades?" in Proc. 2001 IEEE Signal Process. Advances Wireless Commun., pp. 267-270.

[7] C. Tepedelenlioglu, "Maximum multipath diversity with linear equalization in precoded OFDM systems," IEEE Trans. Inf. Theory, vol. 50,pp. 232-235, Jan. 2004.

[8] C. Tepedelenlioglu and Q. Ma, "On the performance of linear equalizers for block transmission systems," in Proc. 2005 IEEE GLOBECOM, vol. 6. MEHANA and NOSRATINIA: SINGLECARRIER FREQUENCY-DOMAIN EQUALIZER WITH MULTI-ANTENNA TRANSMIT DIVERSITY 397

[9] B. Muquet, Z. Wang, G. Giannakis, M. de Courville, and P. Duhamel, "Cyclic prefixing or zero padding for wireless multicarrier transmissions?" IEEE Trans. Commun., vol. 50, no. 12, pp. 2136-2148, Dec. 2002.

[10] Z. Wang and G. Giannakis, "Complex-field coding for OFDM over fading wireless channels," IEEE Trans. Inf. Theory, vol. 49, no. 3, pp. 707-720, Mar. 2003.

[11] H. Gao, P. J. Smith, and M. V. Clark, "Theoretical reliability of MMSE linear diversity combining in Rayleigh-fading additive interference channels," IEEE Trans. Commun., vol. 46, no. 5, pp. 666-672, May 1998.

[12] L. Zheng and D. N. C. Tse, "Diversity and multiplexing: a fundamental tradeoff in multiple-antenna channels," IEEE Trans. Inf. Theory, vol. 49, no. 5, pp. 1073-1096, May 2003

[13] Wittneben, "A new bandwidth efficient transmit antenna modulation diversity scheme for linear digital modulation," in Proc. 1993 IEEE ICC, vol. 3, pp. 1630-1634.

[14] A. Dammann and S. Kaiser, "On the equivalence of space-time block coding with multipath propagation and/or cyclic delay diversity in OFDM," in 2002 IEEE European Wireless.

[15] G. Bauch and J. Malik, "Cyclic delay diversity with bit-interleaved coded modulation in orthogonal frequency division multiple access," IEEE Trans. Wireless Commun., vol. 5, no. 8, pp. 2092 2100, Aug. 2006

[16] U.-K. Kwon and G.-H. Im, "Cyclic delay diversity with frequency domain turbo equalization for uplink fast fading channels," IEEE Commun. Lett., vol. 13, no. 3, pp. 184-186, Mar. 2009.

[17] A. Dammann, "On antenna diversity techniques for OFDM systems," Ph.D. dissertation, Universit" at Ulm, Germany, June 2005, VDI Verlag D“usseldorf, Series 10, No. 766

[18] S. Zhou and G. Giannakis, "Single-carrier space-time block-coded transmissions over frequency-selective fading channels," IEEE Trans. Inf. Theory, vol. 49, no. 1, pp. 164-179, Jan. 2003.

[19] L. Grokop and D. Tse, "Diversity-multiplexing tradeoff in ISI channels," IEEE Trans. Inf. Theory, vol. 55, no. 1, pp. 109-135, Jan. 2009.

[20] A. Hesham Mehana and A. Nosratinia, "Diversity of MMSE MIMO receivers," in Proc. 2010 IEEE ISIT.

\section{BIOGRAPHY}

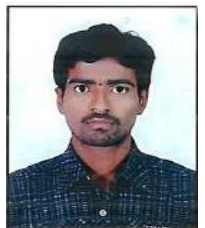

Sunil Shankar Mane received BE from VTU Belgaum and Pursuing ME in Signal processing at Savitribai Phule Pune University India. My area of interest is signal processing and image processing. 